Supporting Information

\title{
Water Chestnut-Derived Slope-Dominated Carbon as a High-Performance Anode for High-Safety Potassium-Ion Batteries
}

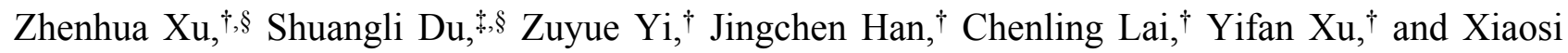
Zhou*,†

$\dagger$ Jiangsu Key Laboratory of New Power Batteries, Jiangsu Collaborative Innovation Center of Biomedical Functional Materials, School of Chemistry and Materials Science, Nanjing Normal University, Nanjing, 210023, China

\$School of Safety and Emergency Management Engineering, Taiyuan University of Technology, Taiyuan, 030024, China

*Corresponding author. E-mail: zhouxiaosi@,njnu.edu.cn 


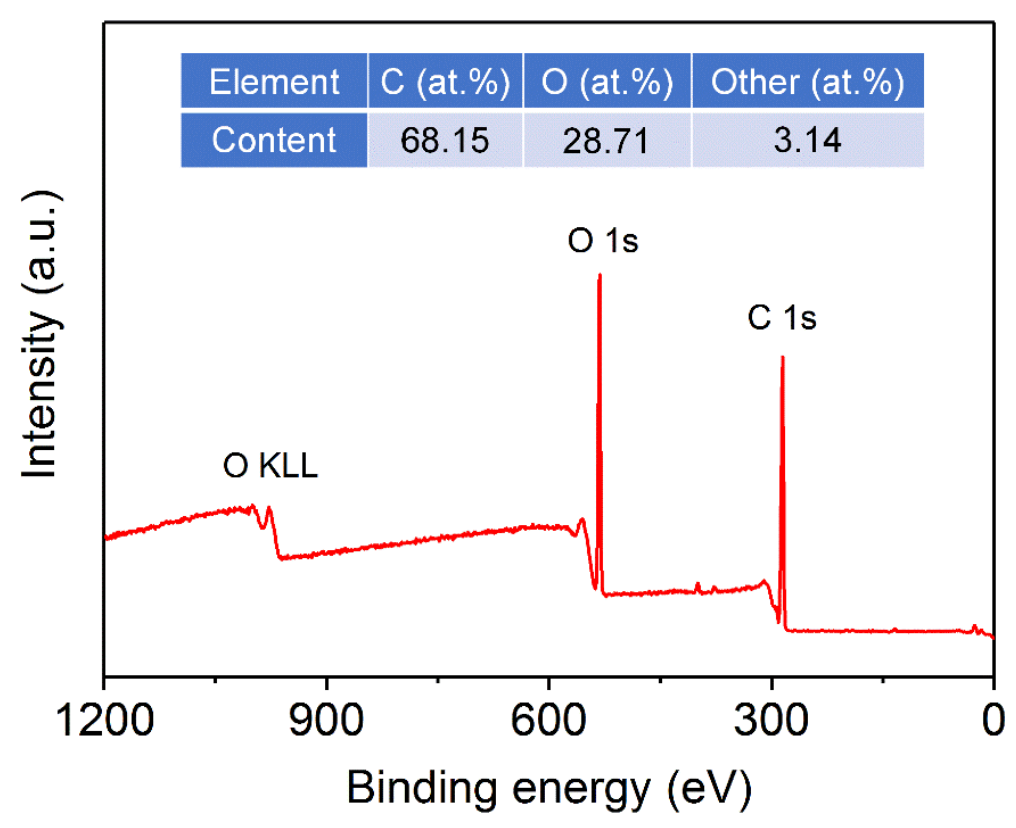

Figure S1. XPS full spectrum of freeze-dried water chestnut.

As illustrated in Figure S1, the oxygen atomic content in the freeze-dried water chestnut is as high as $28.71 \%$. 

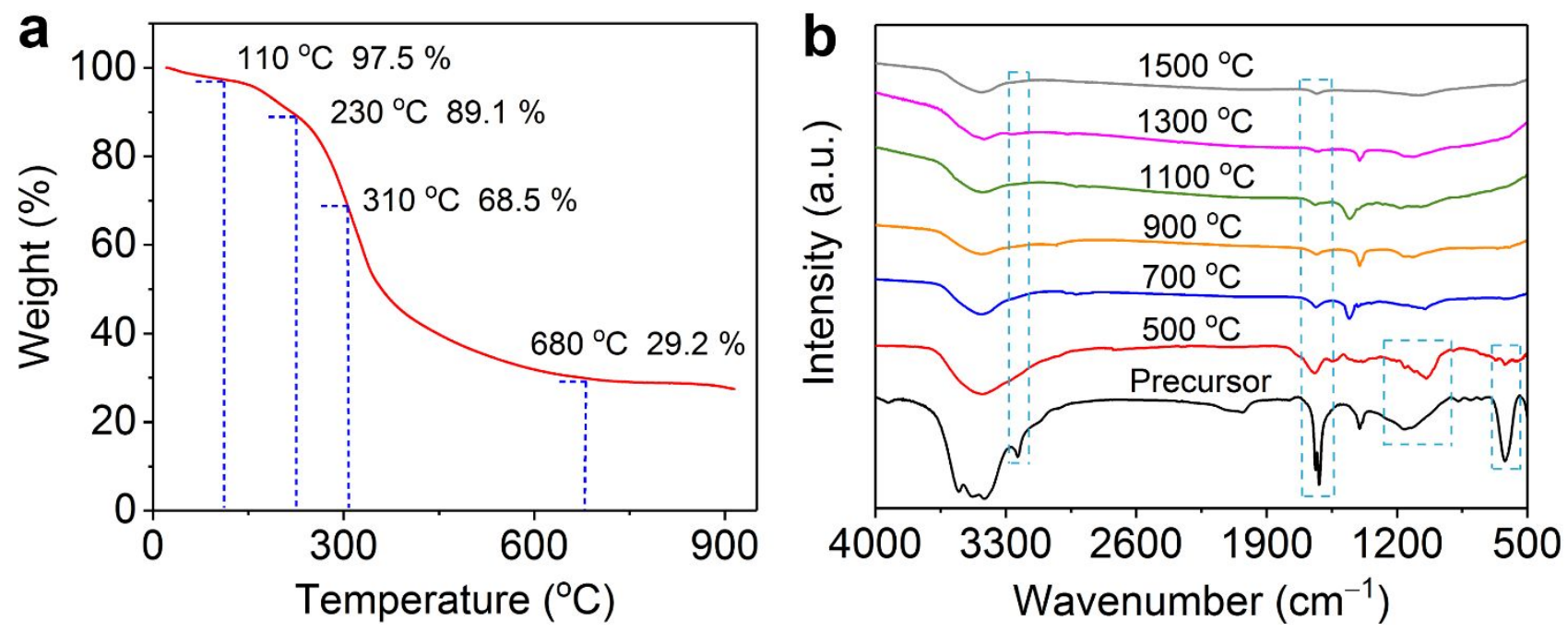

Figure S2. (a) TG curves of the freeze-dried water chestnut. (b) FT-IR spectra of the freeze-dried water chestnut and the water chestnut-derived carbon (denoted as WC) samples obtained at different pyrolysis temperatures.

As can be seen from Figure 2a, there is only slight weight degradation $(2.5 \mathrm{wt} \%)$ before $110{ }^{\circ} \mathrm{C}$, which can be attributed to the loss of adsorbed organic molecules and moisture. At approximately $310^{\circ} \mathrm{C}$, it begins to lose weight apparently. Specifically, it undergoes a dramatic weight loss (39.3 $\mathrm{wt} \%$ ) between 310 and $680{ }^{\circ} \mathrm{C}$. Afterwards, the weight varies slowly as the temperature rises further. The FT-IR spectra in Figure S2b displays a strong O-H peak between 3700 and $3300 \mathrm{~cm}^{-1}$, a C-H vibration band at around $3000 \mathrm{~cm}^{-1}$, and various vibration modes of $\mathrm{C}-\mathrm{C}, \mathrm{C}=\mathrm{C}, \mathrm{C}=\mathrm{O}, \mathrm{C}-\mathrm{O}$ in the range of $1700-500 \mathrm{~cm}^{-1}$. 


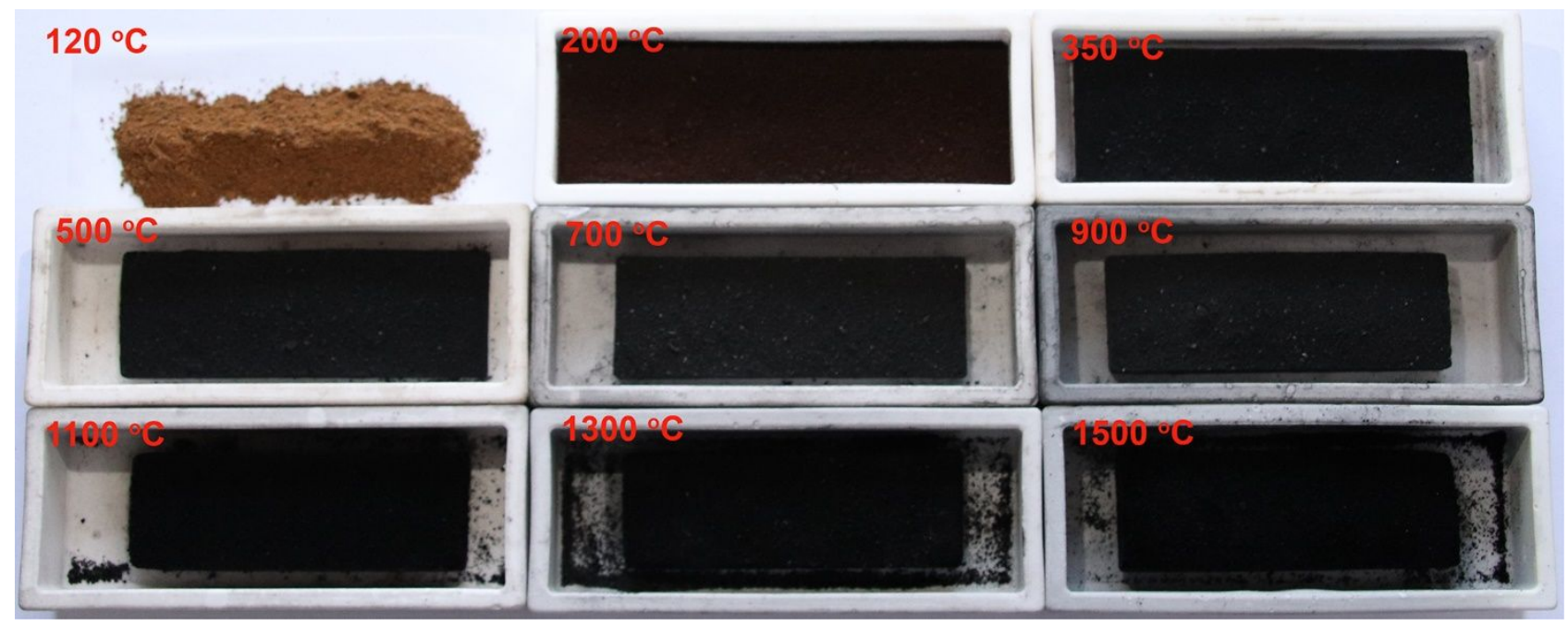

Figure S3. The digital images obtained at different temperatures.

As shown in Figure S3, there is no evident variation from 120 to $200{ }^{\circ} \mathrm{C}$, always maintaining as dark brown powder. In the range of 350 to $500{ }^{\circ} \mathrm{C}$, the water chestnut goes through a series of shrinking, solidifying, and fracturing, accompanied by the release of many small organic molecules. When the pyrolysis temperature is higher than $500{ }^{\circ} \mathrm{C}$, the rearrangement of graphene nanosheets derived from organic molecules is gradually proceeding. 


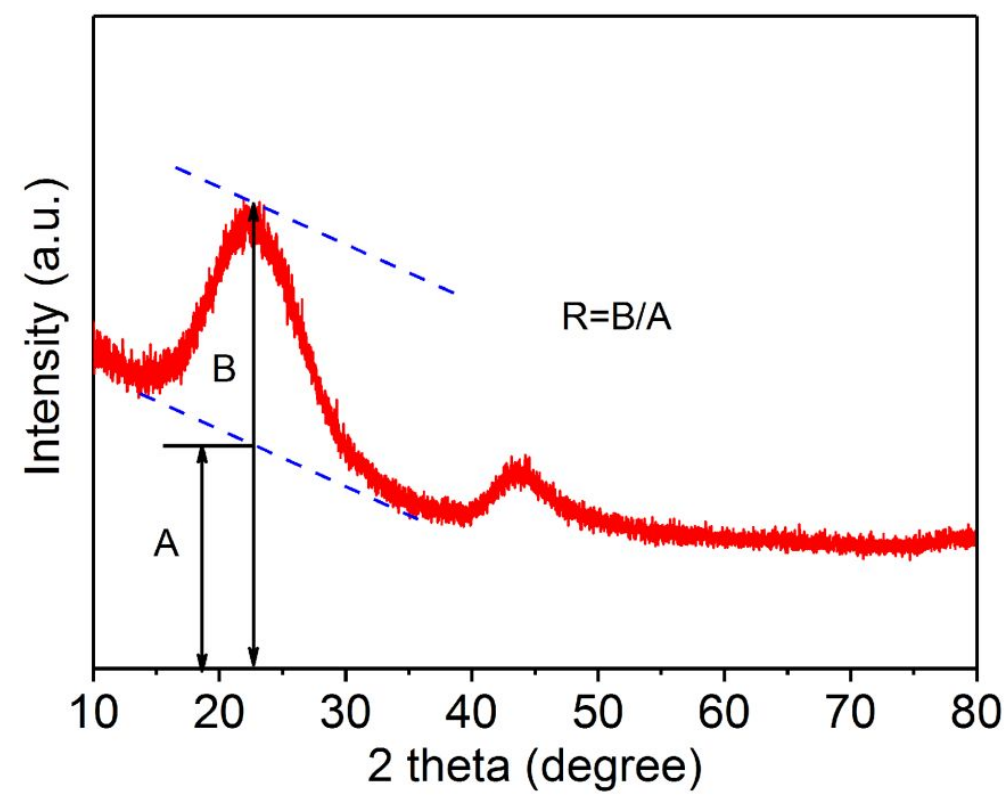

Figure S4. Schematic illustration of the definition of R parameter.

As $\mathrm{R}$ represnts the number of graphene nanosheets arranged as single layer along the $c$ direction, smaller $\mathrm{R}$ value implies the carbon is less-developed in stacking direction and in more disordered state. R-value is based on the ratio of the intensity at the (002) peak (B) and the background (A) at the same angle. 

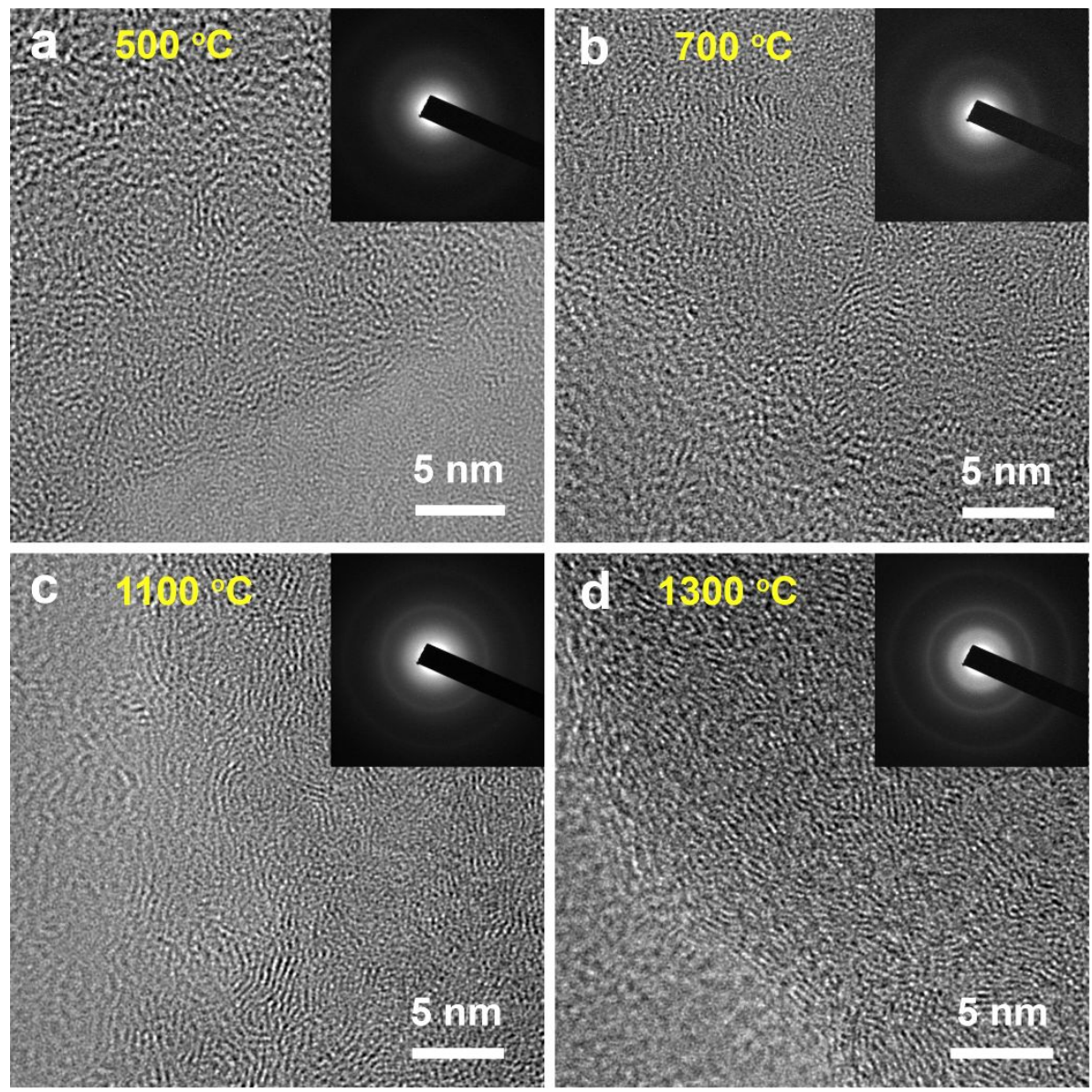

Figure S5. HRTEM and SAED images of WC samples achieved at (a) $500{ }^{\circ} \mathrm{C}$, (b) $700{ }^{\circ} \mathrm{C}$, (c) 1100 ${ }^{\circ} \mathrm{C}$, and (d) $1300{ }^{\circ} \mathrm{C}$. 

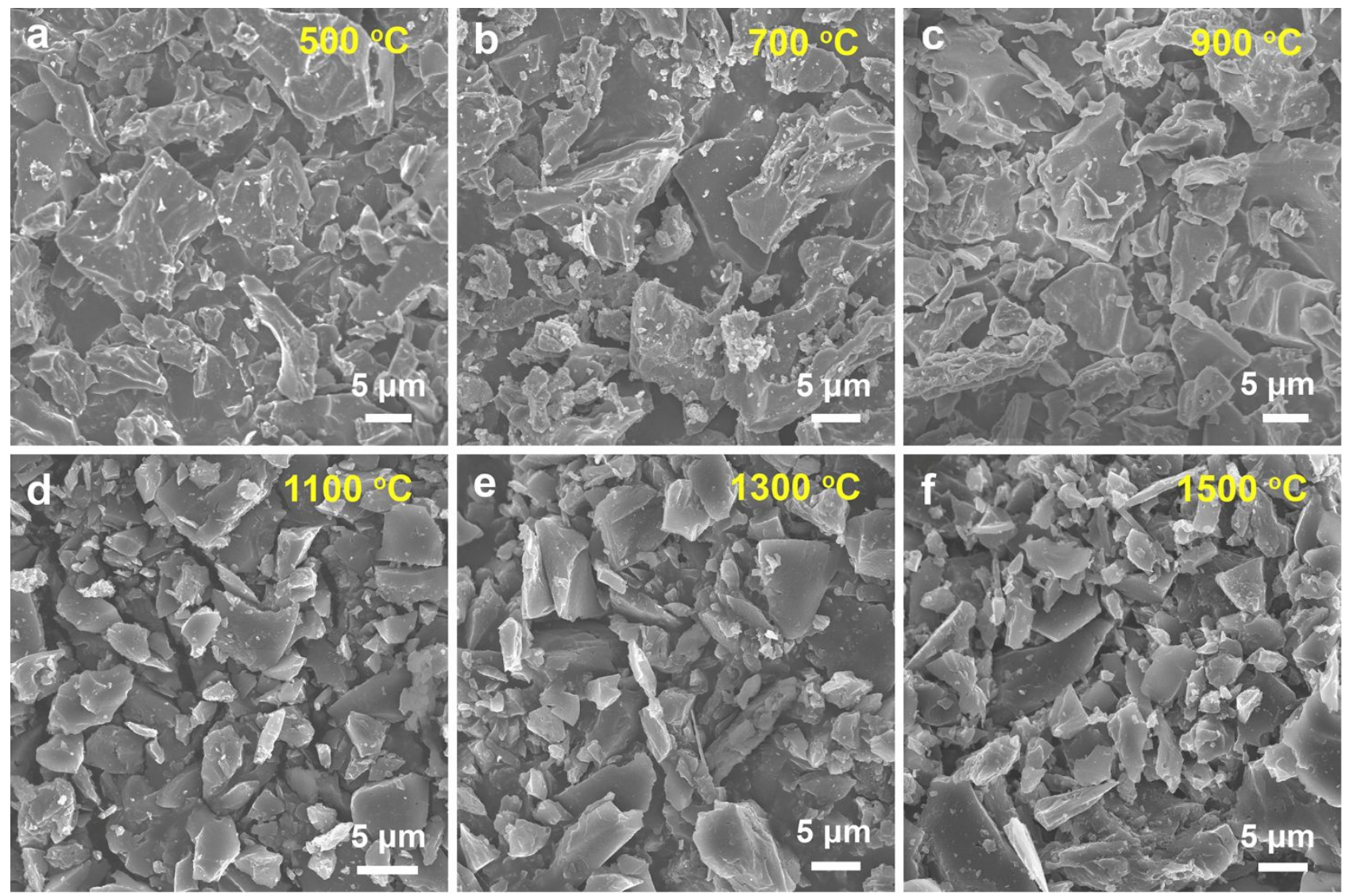

Figure S6. SEM images of WC samples obtained at (a) $500{ }^{\circ} \mathrm{C}$, (b) $700{ }^{\circ} \mathrm{C}$, (c) $900{ }^{\circ} \mathrm{C}$, (d) $1100{ }^{\circ} \mathrm{C}$, (e) $1300{ }^{\circ} \mathrm{C}$, and (f) $1500^{\circ} \mathrm{C}$. 

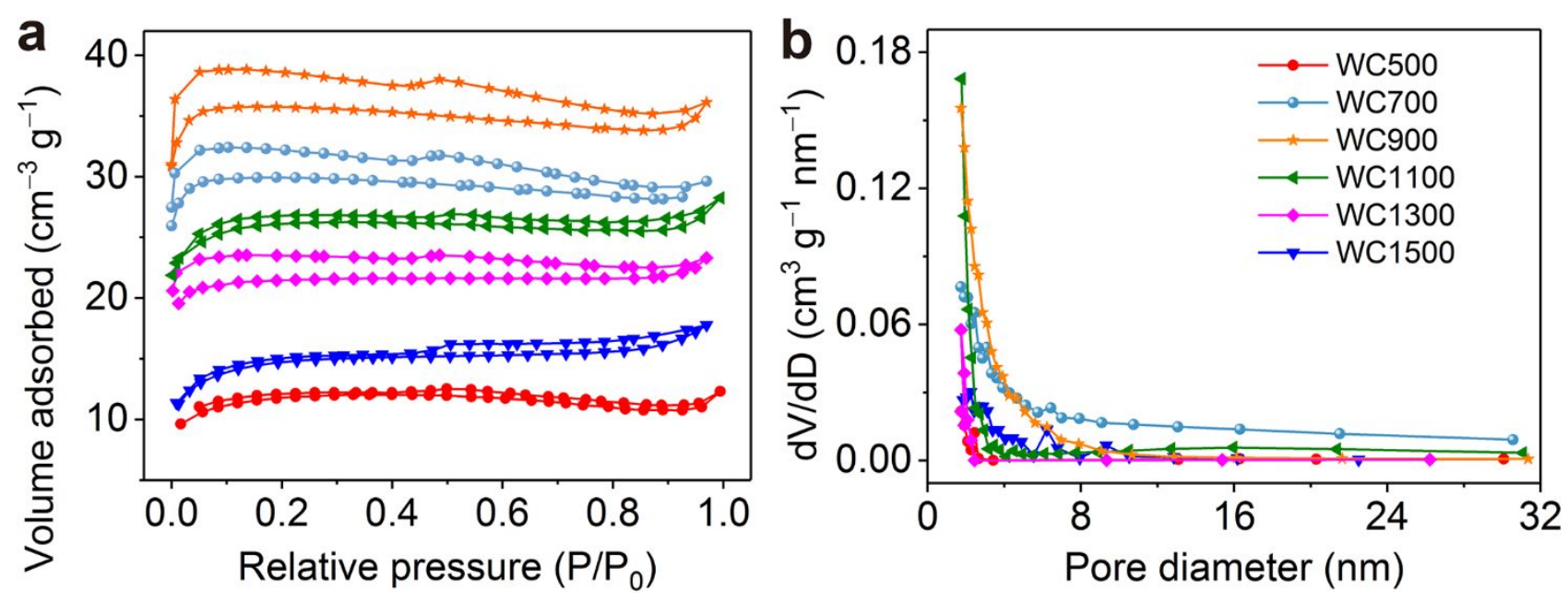

Figure S7. (a) $\mathrm{N}_{2}$ adsorption-desorption isotherms and (b) the corresponding pore size distribution of WC samples produced at diverse temperature.

Nitrogen absorption-desorption isotherm analysis (Figure S7) shows that the Brunauer-Emmett-Teller specific surface area of WC500, WC700, WC900, WC1100, WC1300, and $\mathrm{WC} 1500$ is $36.5,85.8,106.5,79.2,64.8$, and $48.6 \mathrm{~m}^{2} \mathrm{~g}^{-1}$, respectively. 

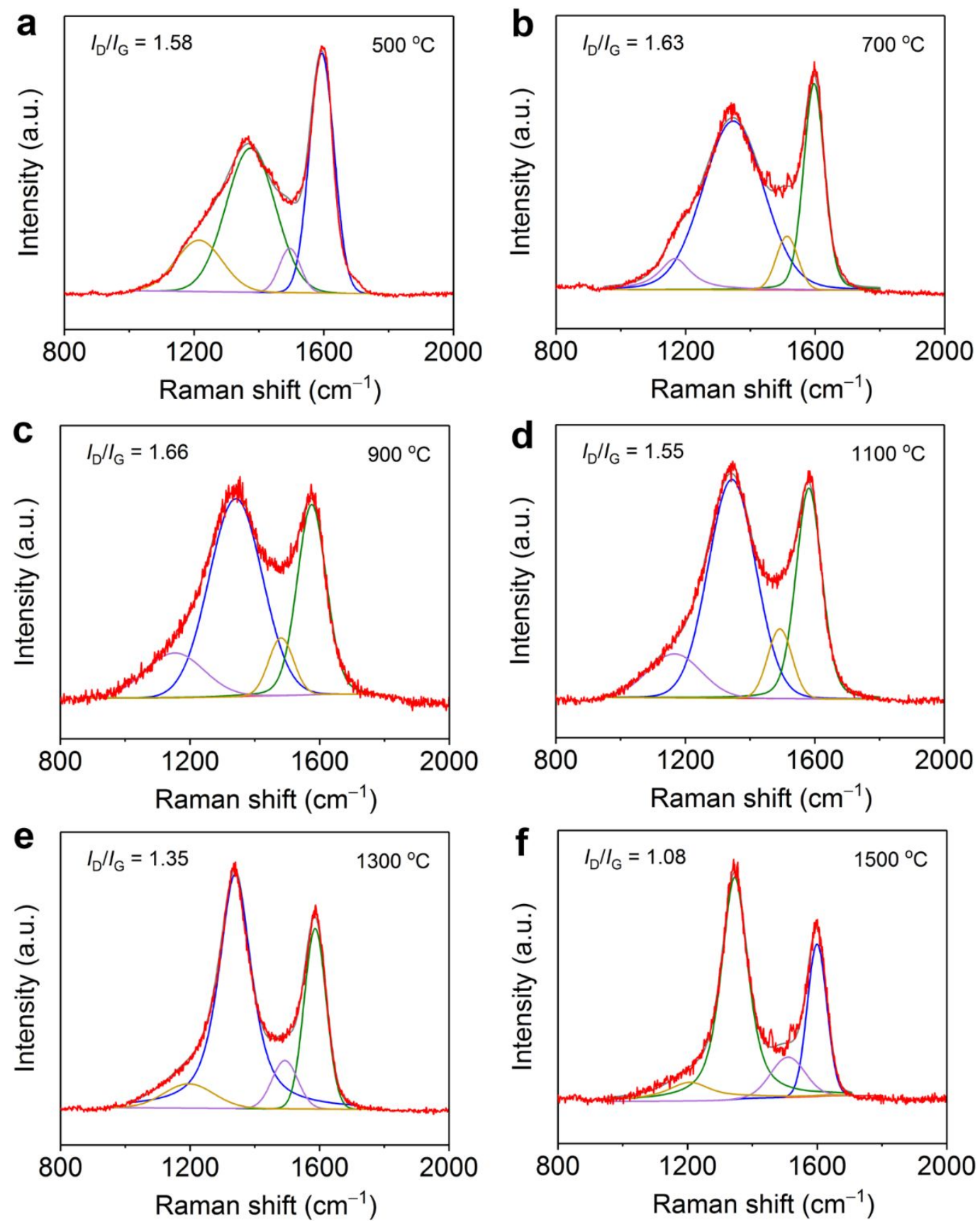

Figure S8. Raman spectra of WC samples synthesized at (a) $500{ }^{\circ} \mathrm{C}$, (b) $700{ }^{\circ} \mathrm{C}$, (c) $900{ }^{\circ} \mathrm{C}$, (d) 1100 ${ }^{\circ} \mathrm{C}$, (e) $1300{ }^{\circ} \mathrm{C}$, and (f) $1500{ }^{\circ} \mathrm{C}$.

$L_{\mathrm{a}}$ can be calculated by Raman spectra according to the following equation:

$$
L_{\mathrm{a}}=\left(2.4 \times 10^{-10}\right) \lambda_{\mathrm{nm}}^{4}\left(\frac{I_{\mathrm{G}}}{I_{\mathrm{D}}}\right)
$$

where $\lambda_{\mathrm{nm}}$ indicates the laser wavelength $(532 \mathrm{~nm}), I_{\mathrm{D}}$ denotes the integrated area of the $\mathrm{D}$ band, and $I_{\mathrm{G}}$ represents the integrated area of the $\mathrm{G}$ band. 


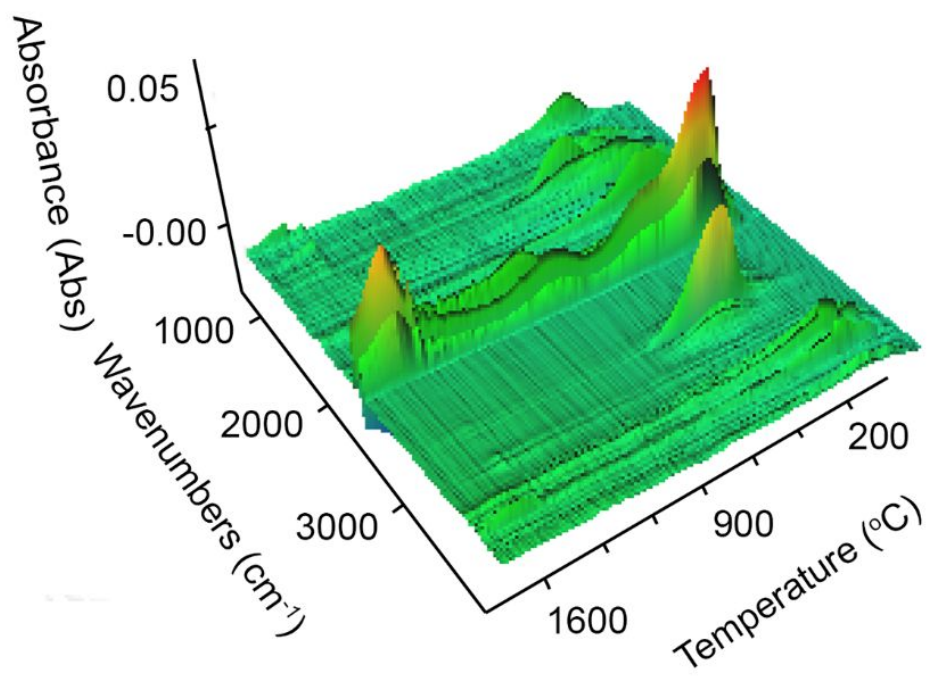

Figure S9. Temperature-dependent IR spectra of the decomposition species from the pyrolysis of the freeze-dried water chestnut.

During carbonization, IR spectra coupled with mass spectroscopy (MS, Figure S9) was applied to identify the chemical species released. Some small molecules with lower oxygen content, such as $\mathrm{CH} \equiv \mathrm{CH}, \mathrm{CH}_{3} \mathrm{OCH}_{3}$, and furfural, are detected before $900{ }^{\circ} \mathrm{C}$ pyrolysis of the freeze-dried water chestnut. The release of molecules with higher oxygen content, such as $\mathrm{CO}_{2}$ and $\mathrm{CO}$ mainly appear after $900{ }^{\circ} \mathrm{C}$. 

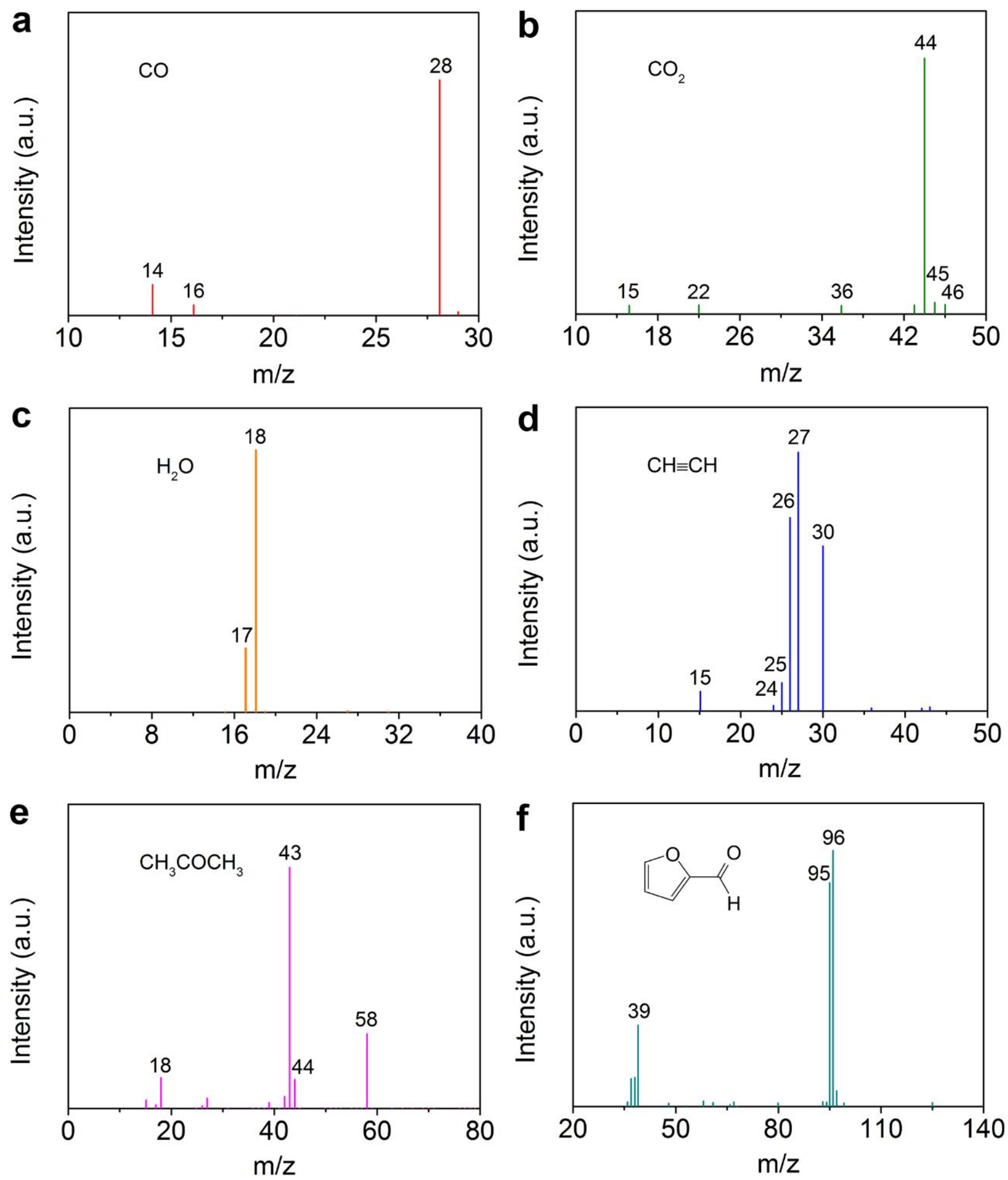

Figure S10. The MS spectra of (a) $\mathrm{CO}$, (b) $\mathrm{CO}_{2}$, (c) $\mathrm{H}_{2} \mathrm{O}$, (d) $\mathrm{CH} \equiv \mathrm{CH}$, (e) $\mathrm{CH}_{3} \mathrm{OCH}_{3}$, and (f) furfural. 


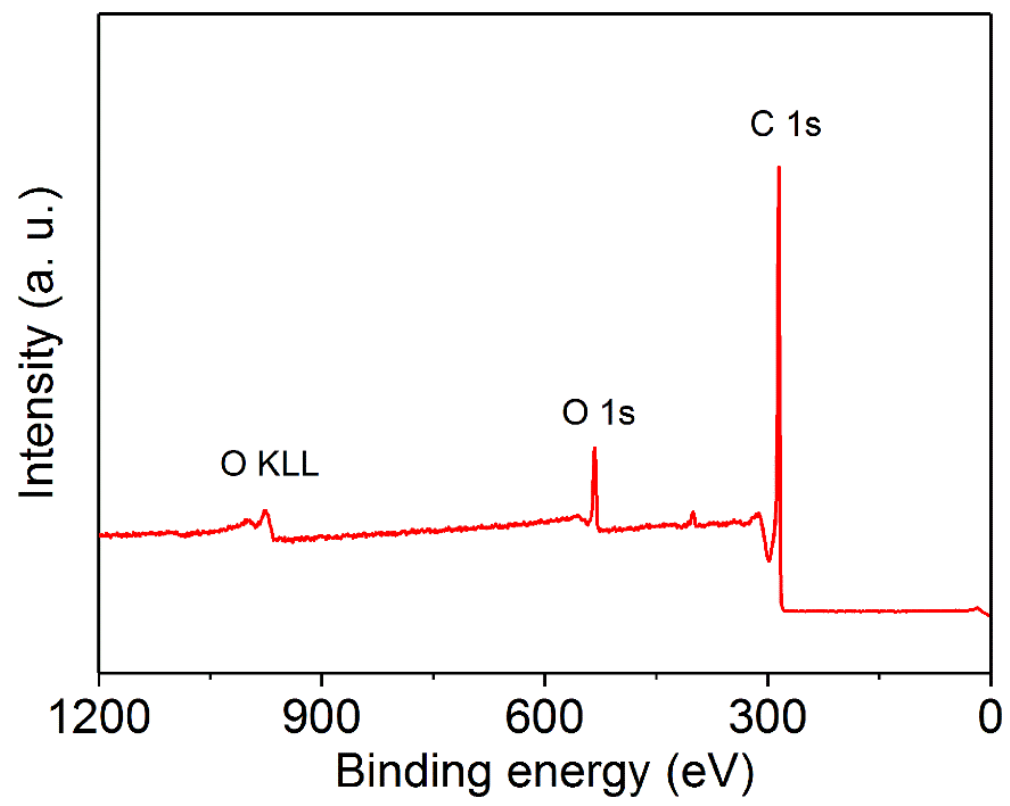

Figure S11. XPS full spectrum of WC900. 

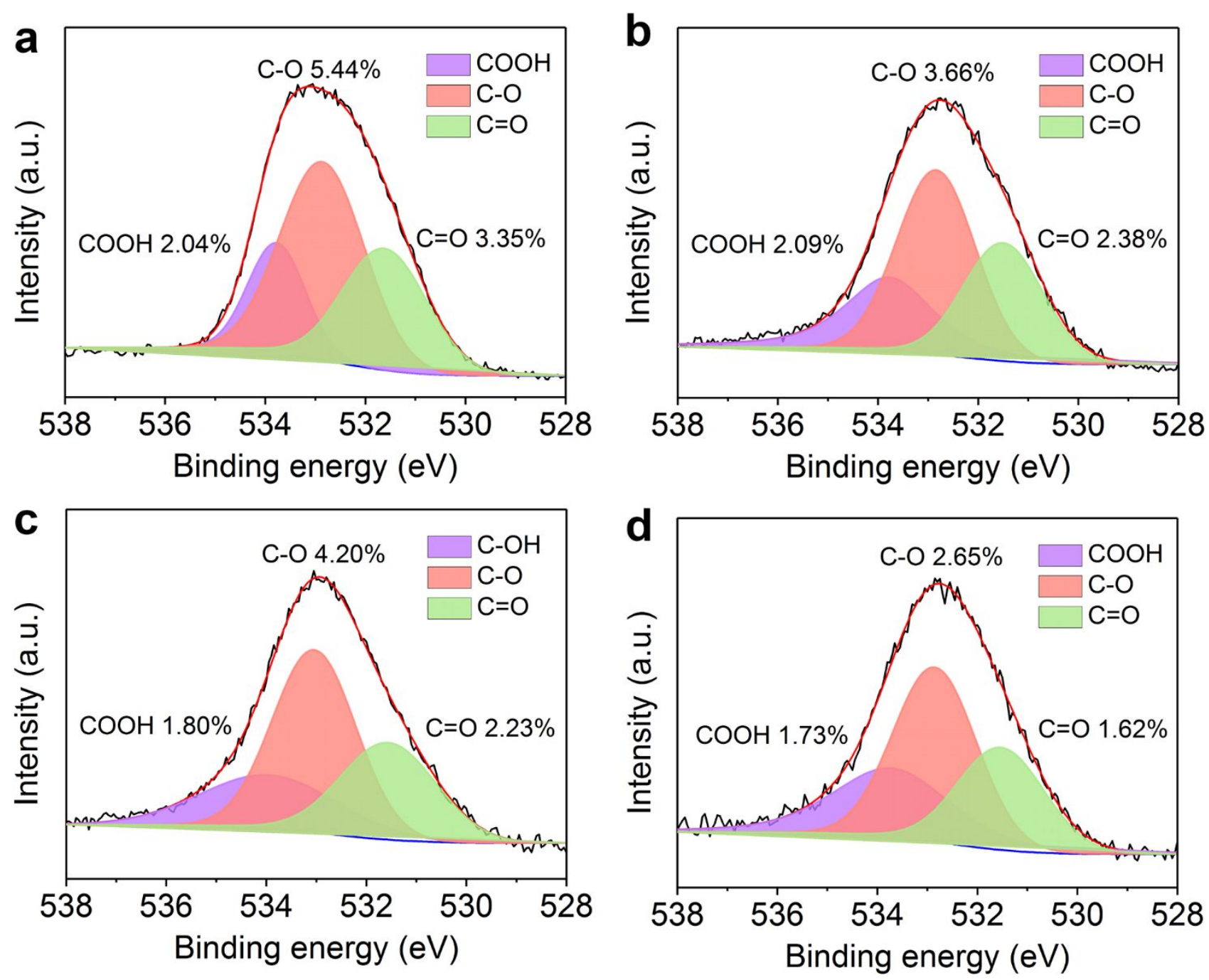

Figure S12. The high-resolution of O 1s XPS peak of (a) WC500, (b) WC700, (c) WC1100, and (d) WC1500. 

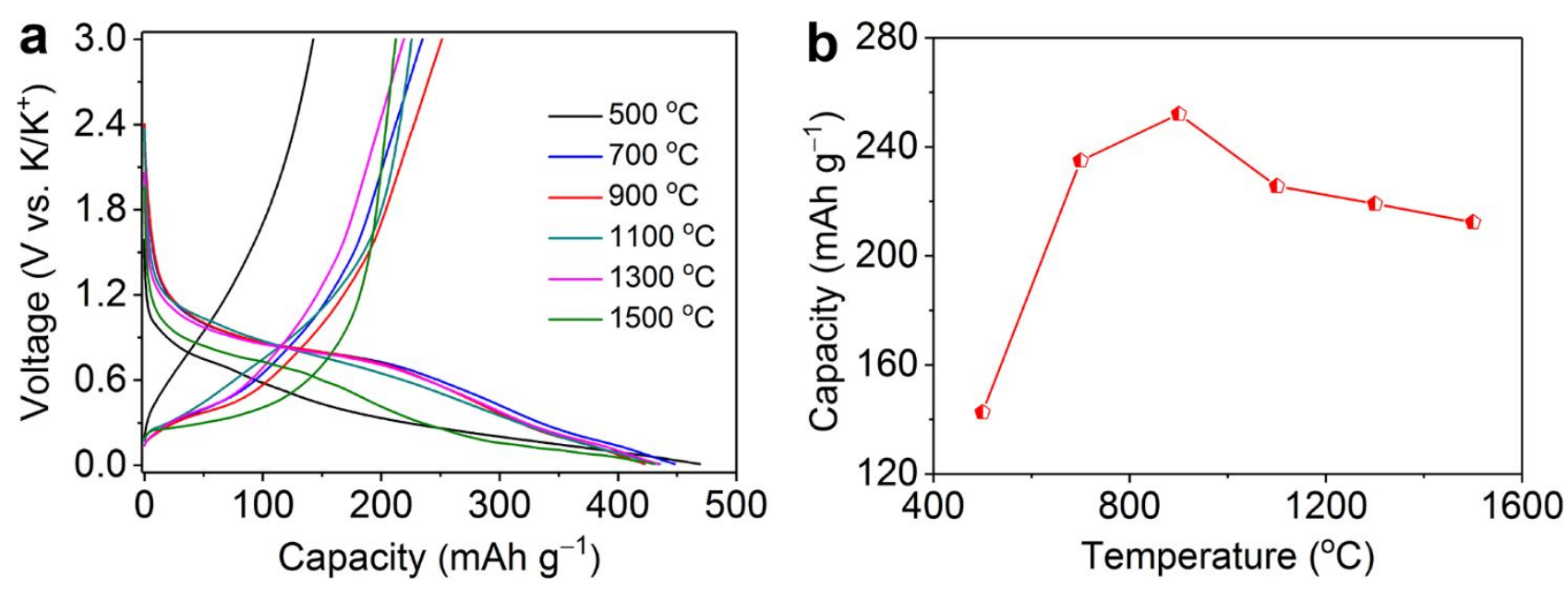

Figure S13. (a) The first discharge/charge curves of the WC samples formed at different temperatures at a current rate of $100 \mathrm{~mA} \mathrm{~g}^{-1}$ between $0.01-3.0 \mathrm{~V}$ and (b) variation of initial reversible capacity with temperatures. 

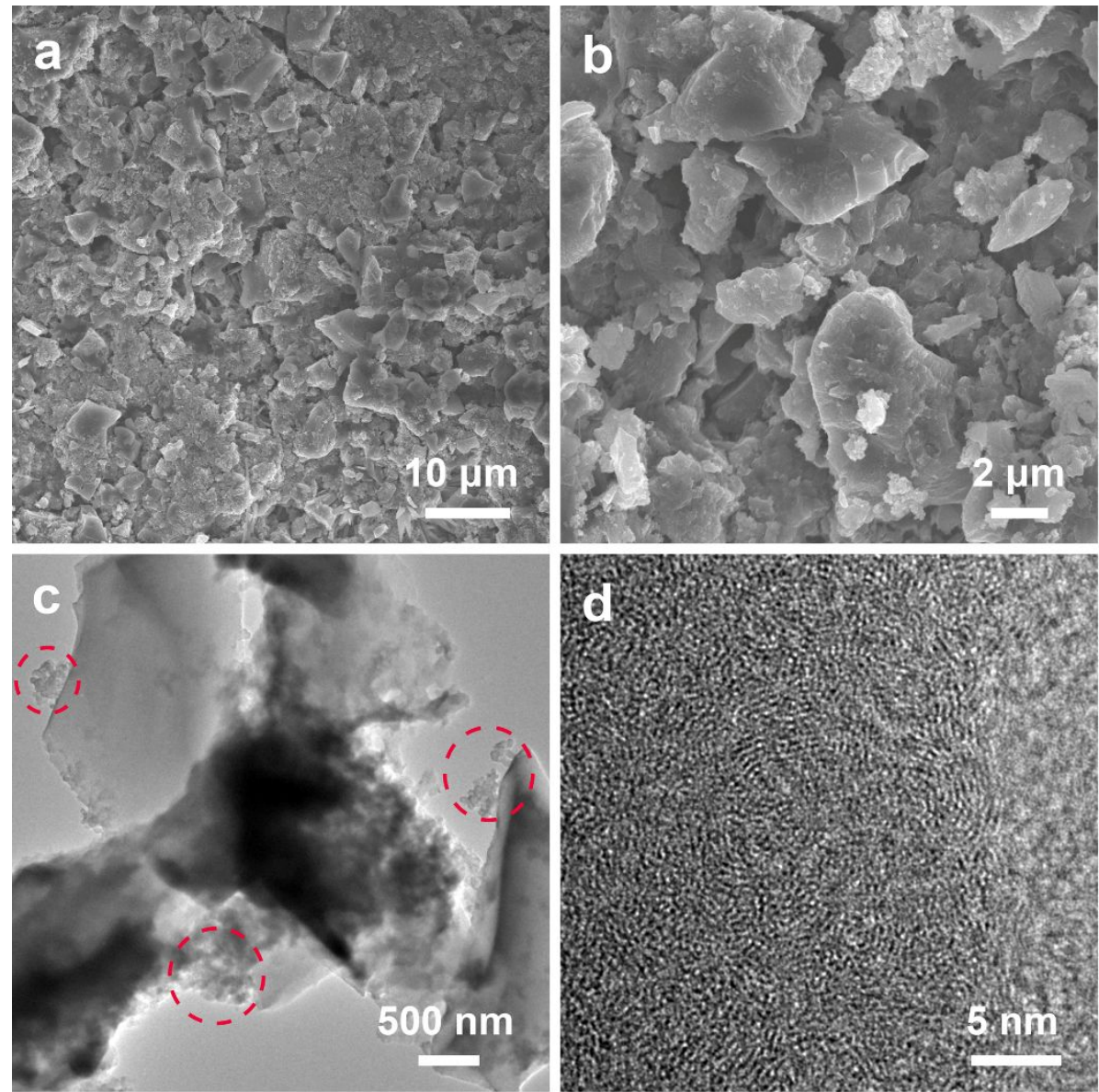

Figure S14. (a, b) SEM, (c) TEM and (d) HRTEM images of the WC900 electrode after 1000 cycles (The nanoparticles in the red circle are Super-P carbon black). 

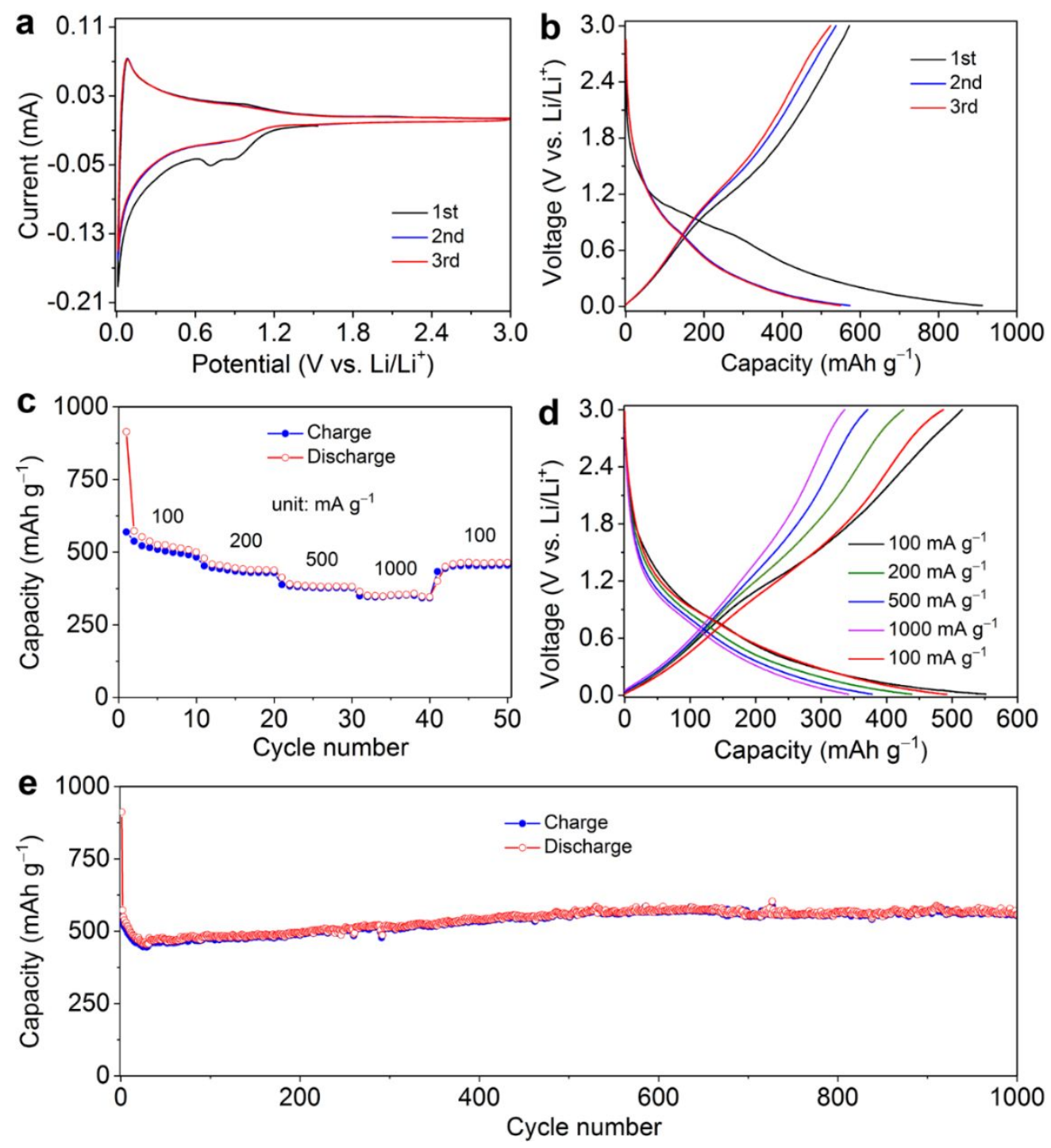

Figure S15. Lithium storage performance of WC900: (a) CV curves for the initial three cycles at a scan rate of $0.1 \mathrm{mV} \mathrm{s}^{-1}$, (b) galvanstatic charge-discharge profiles for the first three cycles at $100 \mathrm{~mA}$ $\mathrm{g}^{-1}$, (c) rate performance, (d) galvanstatic charge-discharge curves at the different current density, and (e) long cycling performance at $100 \mathrm{~mA} \mathrm{~g}^{-1}$.

The electrolyte for lithium-ion batteries (LIBs) is $1.0 \mathrm{M} \mathrm{LiPF} 6$ dissolved in ethylene carbonate/dimethyl carbonate (EC/DEC, 1:1 in volume). When used as an anode material for LIBs, WC900 shows a high initial charge capacity of $571.6 \mathrm{mAh} \mathrm{g}^{-1}$ at a current density of $100 \mathrm{~mA} \mathrm{~g}^{-1}$, remarkable rate capability of $350.3 \mathrm{mAh} \mathrm{g}^{-1}$ at $1000 \mathrm{~mA} \mathrm{~g}^{-1}$, and long cyclic stability of $556.8 \mathrm{mAh}$ $\mathrm{g}^{-1}$ after 1000 cycles at $100 \mathrm{~mA} \mathrm{~g}^{-1}$. 

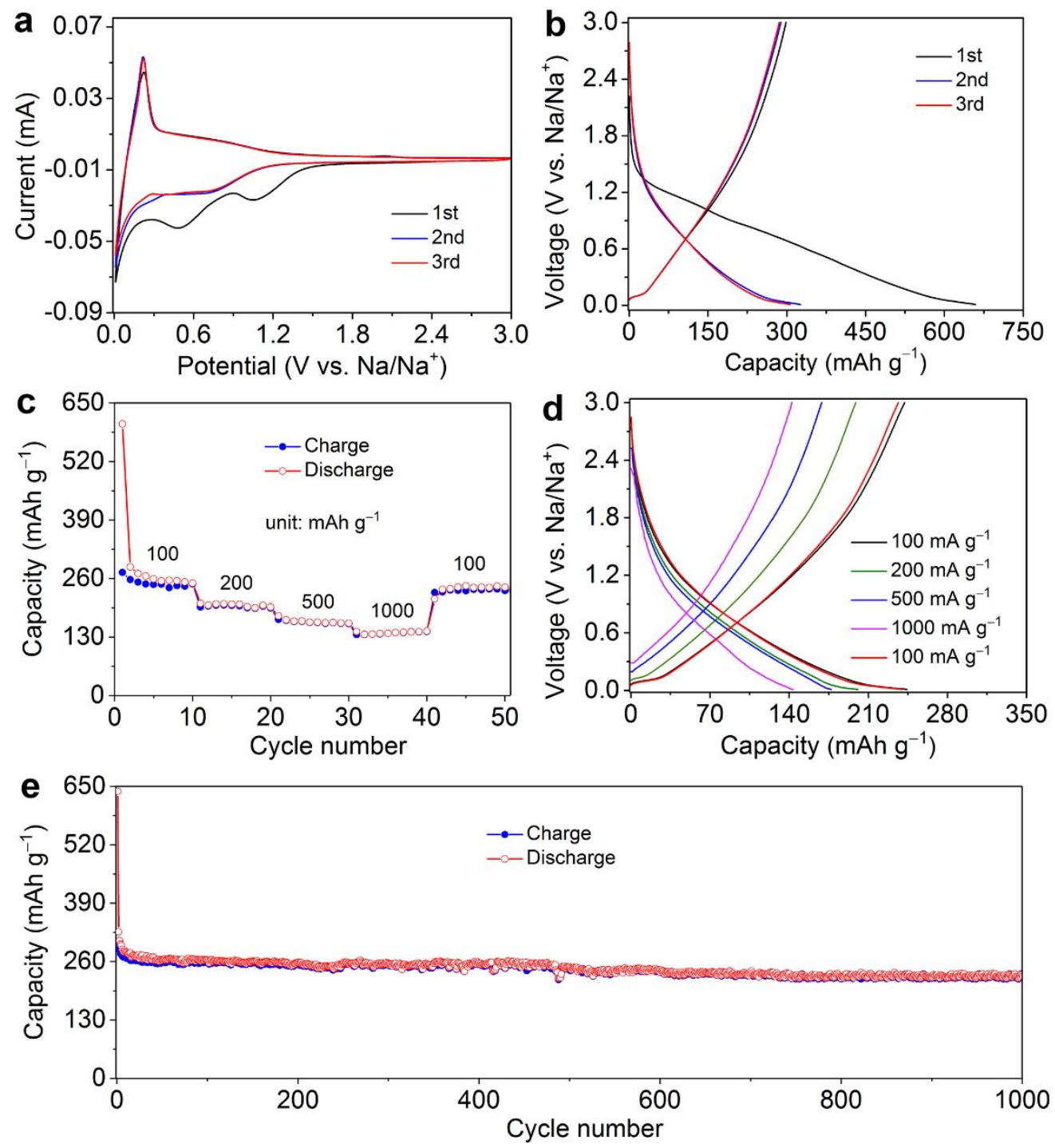

Figure S16. Sodium storage properties of WC900: (a) CV profiles for the first three cycles at a sweep rate of $0.1 \mathrm{mV} \mathrm{s}^{-1}$, (b) galvanstatic charge-discharge curves for the initial three cycles at $100 \mathrm{~mA} \mathrm{~g}^{-1}$, (c) rate capability, (d) galvanstatic charge-discharge profiles at the varied current density, and (e) long cyclic stability at $100 \mathrm{~mA} \mathrm{~g}^{-1}$.

The electrolyte for sodium-ion batteries (SIBs) is $0.8 \mathrm{M} \mathrm{NaClO}_{4}$ in propylene carbonate (PC) with 5\% fluoroethylene carbonate (FEC). When used as an anode material for SIBs, WC900 delivers a high initial reversible capacity of $298.5 \mathrm{mAh} \mathrm{g}^{-1}$ at a current density of $100 \mathrm{~mA} \mathrm{~g}^{-1}$, excellent rate property of $142.6 \mathrm{mAh} \mathrm{g}^{-1}$ at $1000 \mathrm{~mA} \mathrm{~g}^{-1}$, and outstanding cycling performance of $230.8 \mathrm{mAh} \mathrm{g}^{-1}$ after 1000 cycles at $100 \mathrm{~mA} \mathrm{~g}^{-1}$. 

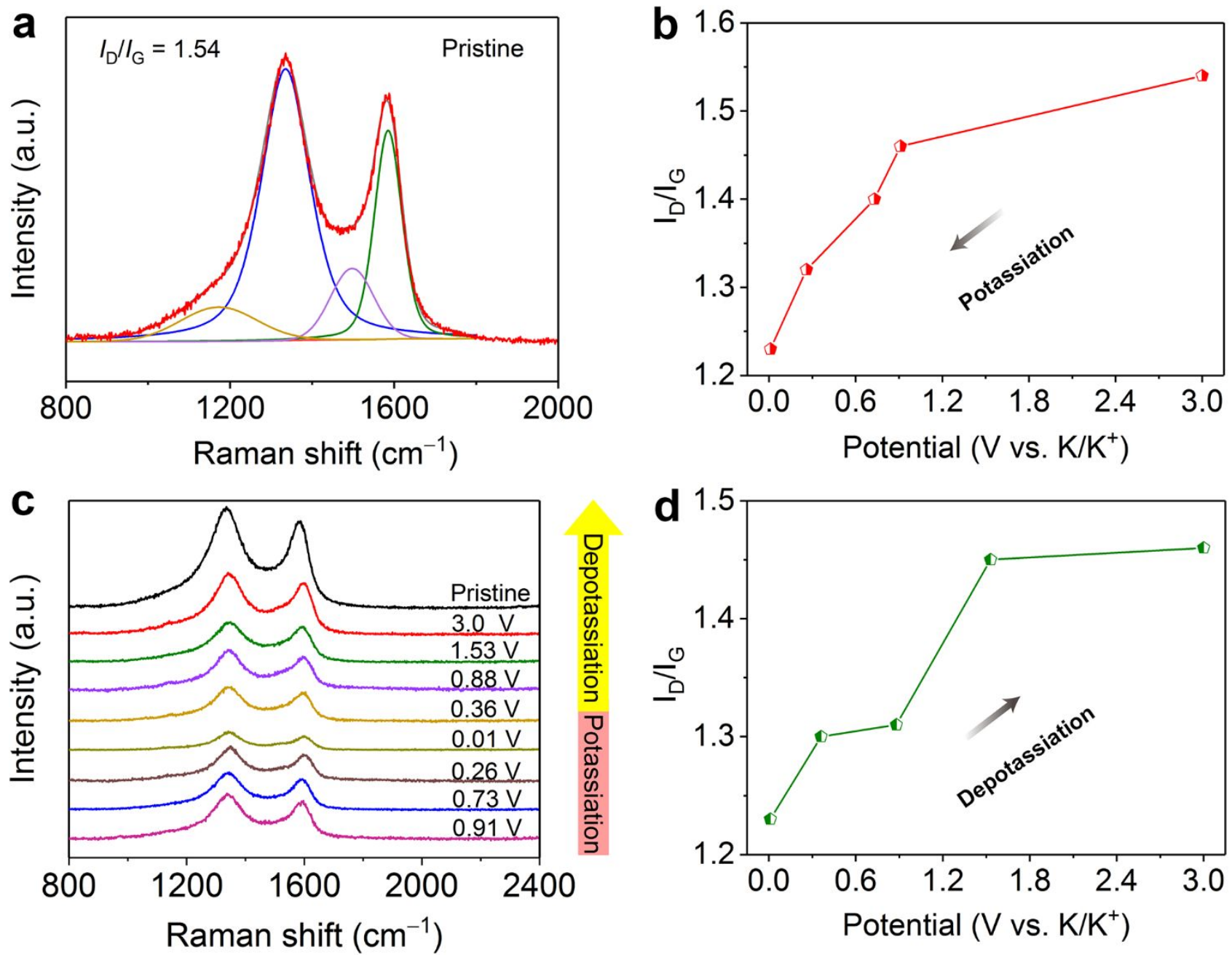

Figure S17. (a) Fitted Raman spectrum of the pristine WC900 electrode. (b) Change in the $I_{\mathrm{D}} / I_{\mathrm{G}}$ with different potassiation states. (c) Ex situ Raman spectra of the WC900 electrodes in PIBs at the different charge and discharge depths. (d) Variation in the $I_{\mathrm{D}} / I_{\mathrm{G}}$ with different depotassiation states. 

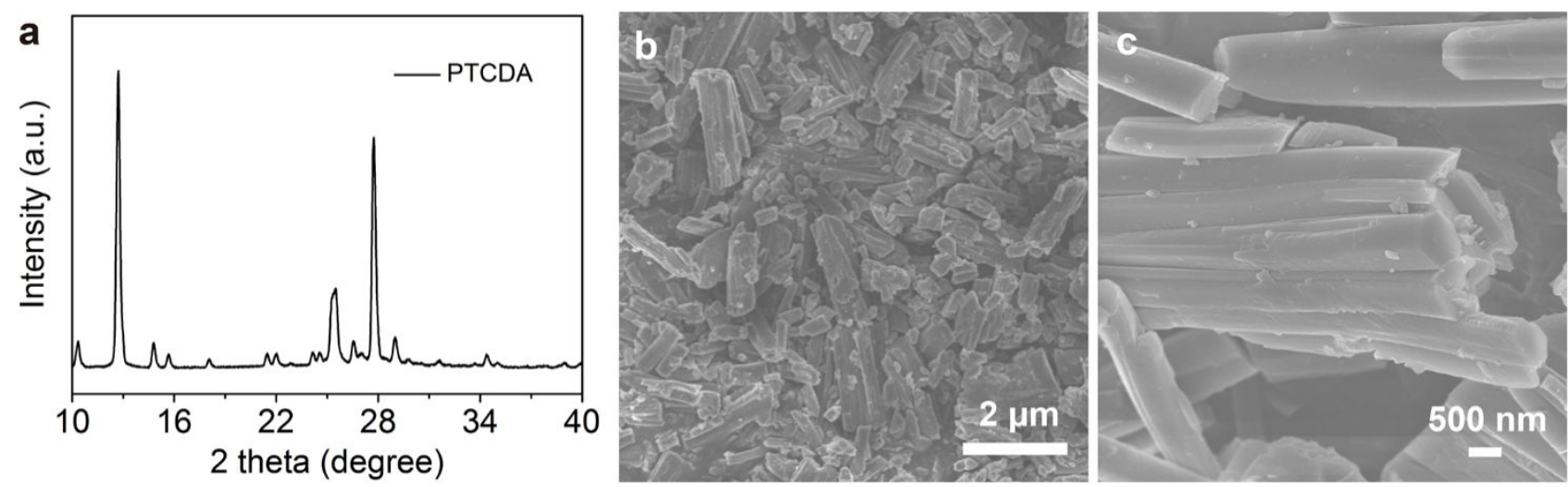

Figure S18. (a) XRD pattern, (b) SEM image, and (c) HRTEM image of commercial PTCDA. 

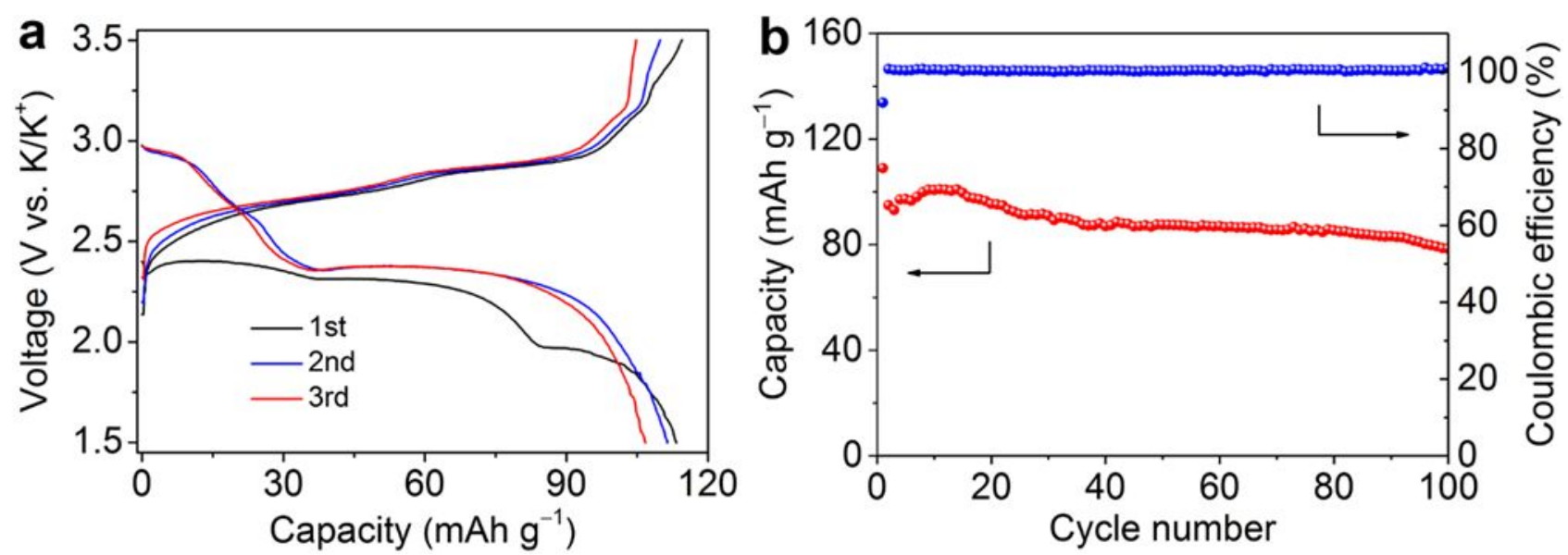

Figure S19. (a) Galvanstatic charge-discharge curves and (b) cycling performance at $100 \mathrm{~mA} \mathrm{~g}^{-1}$ for potassium storage properties of the commercial PTCDA between 1.5 and $3.5 \mathrm{~V}$ at a current density of $100 \mathrm{~mA} \mathrm{~g}^{-1}$. 

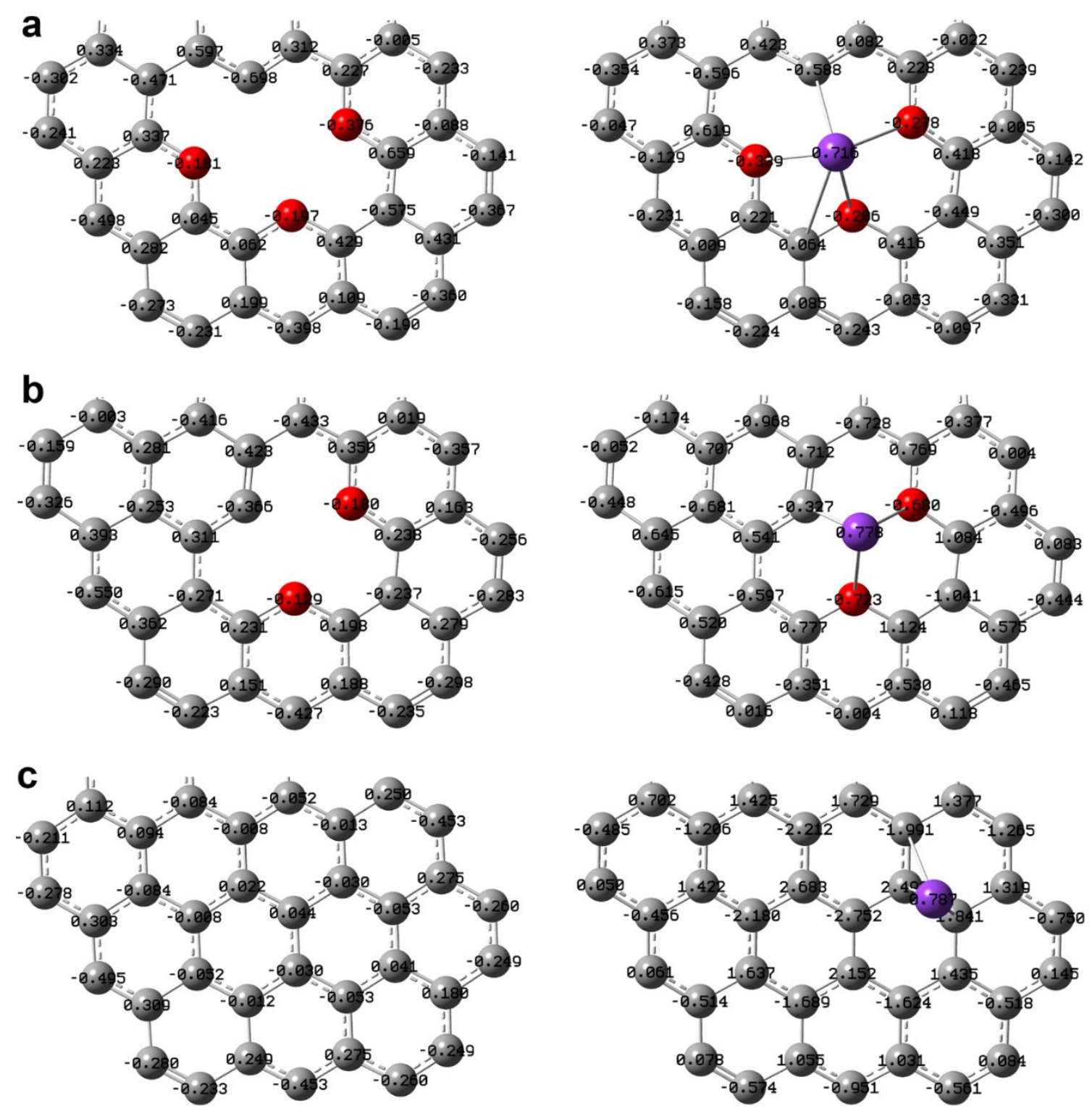

Figure S20. Merz-Kollman (MK) charges of (a) WC900, (b) WC1500, (c) natural graphite (NG) and their corresponding complexes with $\mathrm{K}^{+}$. 
Table S1. Physical parameters obtained from the XRD and Raman measurements.

\begin{tabular}{cccccccc}
\hline Samples & $\mathrm{R}$ & $(002)$ Peak & $\mathrm{d}_{002}(\mathrm{~nm})$ & $L_{\mathrm{c}}(\mathrm{nm})$ & $I_{\mathrm{D}} / I_{\mathrm{G}}$ & $L_{\mathrm{a}}(\mathrm{nm})$ & $\begin{array}{r}\text { BET surface area } \\
\left(\mathrm{m}^{2} \mathrm{~g}^{-1}\right)\end{array}$ \\
\hline WC500 & 1.43 & $22.41^{\circ}$ & 0.396 & 2.23 & 1.58 & 12.17 & 36.5 \\
WC700 & 1.60 & $22.37^{\circ}$ & 0.398 & 2.11 & 1.63 & 11.79 & 85.8 \\
WC900 & 1.96 & $22.39^{\circ}$ & 0.397 & 2.05 & 1.66 & 11.58 & 106.5 \\
WC1100 & 2.54 & $23.40^{\circ}$ & 0.380 & 2.23 & 1.55 & 12.40 & 79.2 \\
WC1300 & 3.17 & $23.71^{\circ}$ & 0.375 & 2.34 & 1.35 & 14.24 & 64.8 \\
WC1500 & 3.87 & $23.87^{\circ}$ & 0.373 & 2.90 & 1.08 & 17.80 & 48.6 \\
\hline
\end{tabular}

Table S2. Elemental analysis for the WC samples synthesized at different temperatures.

\begin{tabular}{cccc}
\hline Samples & C (wt.\%) & O (wt.\%) & Other (wt.\%) \\
\hline WC500 & 90.21 & 1.70 & 8.09 \\
WC700 & 92.37 & 1.50 & 6.13 \\
WC900 & 91.93 & 2.12 & 5.95 \\
WC1100 & 93.46 & 1.36 & 5.18 \\
WC1300 & 95.02 & 0.77 & 4.21 \\
WC1500 & 97.18 & 0.52 & 2.30 \\
\hline
\end{tabular}


Table S3. Elemental content analysis through the XPS spectra.

\begin{tabular}{cccc}
\hline Samples & C (at.\%) & O (at.\%) & Other (at.\%) \\
\hline WC500 & 86.08 & 10.84 & 3.08 \\
WC700 & 88.80 & 8.13 & 3.06 \\
WC900 & 87.44 & 9.30 & 3.26 \\
WC1100 & 89.20 & 8.21 & 2.59 \\
WC1300 & 90.28 & 7.73 & 1.99 \\
WC1500 & 93.68 & 6.00 & 1.32 \\
\hline
\end{tabular}


Table S4. Comparison of the electrochemical performance of different carbon anodes.

\begin{tabular}{|c|c|c|c|c|}
\hline Samples & $\begin{array}{l}1^{\text {st }} \text { reversible capacity } \\
\quad\left(\mathrm{mAh} \mathrm{g}^{-1}\right)\end{array}$ & Cycling stability & $\begin{array}{l}\text { Rate capability } \\
\qquad\left(\mathrm{mAh} \mathrm{g}^{-1}\right)\end{array}$ & Reference \\
\hline WC900 & $253\left(100 \mathrm{~mA} \mathrm{~g}^{-1}\right)$ & $87.1 \%$ (1000 cycles) & $135\left(1 \mathrm{~A} \mathrm{~g}^{-1}\right)$ & This work \\
\hline $\mathrm{CNC}$ & $212\left(55.8 \mathrm{~mA} \mathrm{~g}^{-1}\right)$ & $92.0 \%$ (100 cycles) & $40\left(1.4 \mathrm{~A} \mathrm{~g}^{-1}\right)$ & 1 \\
\hline HINCA & $340\left(28 \mathrm{~mA} \mathrm{~g}^{-1}\right)$ & $\sim 100 \%$ (150 cycles $)$ & $\begin{array}{c}130(0.56 \mathrm{~A} \\
\left.\mathrm{g}^{-1}\right)\end{array}$ & 2 \\
\hline HCNT & $232(100 \mathrm{~mA} \mathrm{~g}-1)$ & $90.0 \%$ (500 cycles) & $162\left(1.6 \mathrm{~A} \mathrm{~g}^{-1}\right)$ & 3 \\
\hline DS & $243\left(50 \mathrm{~mA} \mathrm{~g}^{-1}\right)$ & $91.0 \%$ (100 cycles) & $126\left(1 \mathrm{~A} \mathrm{~g}^{-1}\right)$ & 4 \\
\hline HCS-SC & $215.1\left(279 \mathrm{~mA} \mathrm{~g}^{-1}\right)$ & $93.0 \%$ (200 cycles) & $81\left(2.8 \mathrm{~A} \mathrm{~g}^{-1}\right)$ & 5 \\
\hline $\mathrm{HCNs}$ & $241.2\left(28 \mathrm{~mA} \mathrm{~g}^{-1}\right)$ & $91.0 \%$ (100 cycles) & $109\left(1.4 \mathrm{~A} \mathrm{~g}^{-1}\right)$ & 6 \\
\hline $\mathrm{S}-\mathrm{BC}$ & $339.3\left(50 \mathrm{~mA} \mathrm{~g}^{-1}\right)$ & Not reported & $124.2\left(1 \mathrm{~A} \mathrm{~g} \mathrm{~g}^{-1}\right)$ & 7 \\
\hline NCSCNT & $323\left(20 \mathrm{~mA} \mathrm{~g}^{-1}\right)$ & $73.1 \%$ (100 cycles) & $75\left(1 \mathrm{~A} \mathrm{~g}^{-1}\right)$ & 8 \\
\hline NOPC & $266\left(50 \mathrm{~mA} \mathrm{~g}^{-1}\right)$ & $76.0 \%$ (100 cycles $)$ & $180\left(1 \mathrm{~A} \mathrm{~g}^{-1}\right)$ & 9 \\
\hline OPDMC & $224\left(30 \mathrm{~mA} \mathrm{~g}^{-1}\right)$ & $93.1 \%$ (100 cycles) & $102\left(1 \mathrm{~A} \mathrm{~g}^{-1}\right)$ & 10 \\
\hline PBPC & $264\left(100 \mathrm{~mA} \mathrm{~g}^{-1}\right)$ & $91.7 \%$ (100 cycles) & $152\left(1 \mathrm{~A} \mathrm{~g}^{-1}\right)$ & 11 \\
\hline $\mathrm{CBC}$ & $231\left(100 \mathrm{~mA} \mathrm{~g}^{-1}\right)$ & $89.1 \%$ (100 cycles) & $135.3\left(1 \mathrm{~A} \mathrm{~g}^{-1}\right)$ & 12 \\
\hline NCS-600 & $224\left(100 \mathrm{~mA} \mathrm{~g}^{-1}\right)$ & $54.0 \%$ (100 cycles $)$ & $66\left(1 \mathrm{~A} \mathrm{~g} \mathrm{~g}^{-1}\right)$ & 13 \\
\hline LPG & $160\left(100 \mathrm{~mA} \mathrm{~g}^{-1}\right)$ & $96.9 \%$ (200 cycles $)$ & $70\left(0.2 \mathrm{~A} \mathrm{~g}^{-1}\right)$ & 14 \\
\hline
\end{tabular}

\section{Note:}

CNC: graphitic carbon nanocage

HINCA: hollow interconnected neuron-like carbon architecture

HCNT: hierarchical carbon nanotube

DS: dandelion seed 
HCS-SC: hard-soft composite carbon

HCNs: hollow carbon nanospheres

S-BC: sulfur-doped bamboo charcoal

NCSCNT: nitrogen-doped cup-stacked carbon nanotube

NOPC: nitrogen/oxygen co-doped hierarchically porous carbon

OPDMC: orange peel-derived mesoporous carbon

PBPC: potato biomass porous carbon

CBC: corn husk-derived biomorphic carbon

NCS-600: N-doped carbon spheres heat-treated at $600{ }^{\circ} \mathrm{C}$

LPG: loofah-derived pseudo-graphite

Table S5. The fitted impedance values $\left(R_{\mathrm{s}}\right.$ and $\left.R_{\mathrm{ct}}\right)$, Warburg factors $\left(\sigma_{\mathrm{w}}\right)$, and $\mathrm{K}^{+}$diffusion coefficients of $\left(D_{\mathrm{K}+}\right)$ of the WC700, WC900, and WC1500 electrodes.

\begin{tabular}{ccccc}
\hline Electrode & $R_{\mathrm{S}}(\Omega)$ & $R_{\mathrm{ct}}(\Omega)$ & $\sigma_{\mathrm{w}}\left(\Omega \mathrm{s}^{-1 / 2}\right)$ & $D_{\mathrm{K}}^{+}\left(\mathrm{cm}^{2} \mathrm{~s}^{-1}\right)$ \\
\hline WC700 & 5.2 & 2558 & 2540 & $2.91 \times 10^{-13}$ \\
WC900 & 3.4 & 2351 & 1560 & $6.39 \times 10^{-13}$ \\
WC1500 & 2.8 & 1926 & 2742 & $2.22 \times 10^{-13}$ \\
\hline
\end{tabular}




\section{References}

(1) Cao, B.; Zhang, Q.; Liu, H.; Xu, B.; Zhang, S.; Zhou, T.; J.; Mao, Pang, W.K.; Guo, Z.; Li, A.; Zhou, J.; Chen, X.; Song, H. Graphitic Carbon Nanocage as a Stable and High Power Anode for Potassium-Ion Batteries, Adv. Energy Mater. 2018, 8, 1801149.

(2) Bin, D.-S.; Lin, X.-J.; Sun, Y.-G.; Xu, Y.-S.; Zhang, K.; Cao, A.M.; Wan, L.-J. Engineering Hollow Carbon Architecture for High-Performance K-Ion Battery Anode, J. Am. Chem. Soc. 2018, $140,7127-7134$.

(3) Wang, Y.; Wang, Z.; Chen, Y.; Zhang, H.; Yousaf, M.; Wu, H.; Zou, M.; Cao, A.; Han, R. P. S. Hyperporous Sponge Interconnected by Hierarchical Carbon Nanotubes as a High-Performance Potassium-Ion Battery Anode, Adv. Mater. 2018, 30, 1802074.

(4) Wang, X.; Zhao, J.; Yao, D.; Xu, Y.; Xu, P.; Chea, Y.; Chen, Y.; Zhu , K.; Cheng, K.; Ye, K.; Yan, J.; Cao, D.; Wang, G. Bio-derived hierarchically porous heteroatoms doped-carbon as anode for high performance potassium-ion batteries, J. Electroanal. Chem. 2020, 871, 114272.

(5) Jian, Z.; Hwang, S.; Li, Z.; Hernandez, A.S.; Wang, X.; Xing, Z.; Su, D.; Ji, X. Hard-Soft Composite Carbon as a Long-Cycling and High-Rate Anode for Potassium-Ion Batteries, Adv. Funct. Mater. 2017, 27, 1700324.

(6) Tao, X.-S.; Sun, Y.-G.; Liu, Y.; Chang, B.-B.; Liu, C.-T.; Xu, Y.-S.; Yang, X.-C.; Cao, A.-M. Facile Synthesis of Hollow Carbon Nanospheres and their Potential as Stable Anode Materials in Potassium-Ion Batteries, ACS Appl. Mater. Interfaces. 2020, 12, 13182-13188.

(7) Tian, S.; Guan, D.; Lu, J.; Zhang, Y.; Liu, T.; Zhao, X.; Yang, C.; Nan, J. Synthesis of the Electrochemically Stable Sulfur-Doped Bamboo Charcoal as the Anode Material of Potassium-Ion Batteries, J. Power Sources 2020, 448, 227572.

(8) Zhao, X.; Tang, Y.; Ni, C.; Wang, J.; Star, A.; Xu, Y. Free-Standing Nitrogen-Doped Cup-Stacked Carbon Nanotube Mats for Potassium-Ion Battery Anodes, ACS Appl. Energy Mater. 2018, 1, $1703-1707$.

(9) Sun, Y.; Xiao, H.; Li, H.; He, Y.; Zhang, Y.; Hu, Y.; Ju, Z.; Zhuang, Q.; Cui, Y. Nitrogen/Oxygen Co-Doped Hierarchically Porous Carbon for High-Performance Potassium Storage, Chem. Eur. J. 2019, 25, 7359-7365. 
(10) Verma, R.; Singhbabu, Y.N.; Didwal, P.N.; Nguyen, A.-G.; Kim, J.; Park, C.-J. Biowaste Orange Peel-Derived Mesoporous Carbon as a Cost-Effective Anode Material with Ultra-Stable Cyclability for Potassium-Ion Batteries, Batteries Supercaps 2020, 3, 1-14.

(11) Cao, W.; Zhang, E.; Wang, J.; Liu, Z.; Ge, J.; Yu, X.; Yang, H.; Lu, B. Potato Dderived Biomass Porous Carbon as Anode for Potassium Ion Batteries, Electrochim. Acta. 2019, 293, 364-370.

(12) Wang, Q.; Gao, C.; Zhang, W.; Luo, S.; Zhou, M.; Liu, Y.; Liu, R.; Zhang, Y.; Wang, Z.; Hao, A. Biomorphic Carbon Derived from Corn Husk as a Promising Anode Materials for Potassium Ion Battery, Electrochim. Acta. 2019, 324, 134902.

(13) Wang, S.; Li, Y.; Ma, F.; Wu, X.; Zhou, P.; Miao, Z.; Gao, P.; Zhuo, S.; Zhou, J. Phenolic ResinBased Carbon Microspheres for Potassium Ion Storage, Appl. Surf. Sci. 2020, 506, 144805.

(14) Wu, Z.; Wang, L.; Huang, J.; Zou, J.; Chen, S.; Cheng, H.; Jiang, C.; Gao, P.; Niu, X. LoofahDerived Carbon as an Anode Material for Potassium Ion and Lithium Ion Batteries, Electrochim. Acta. 2019, 306, 446-453. 